\title{
Zum Verhalten der konformen Abbildung am Rande*).
}

\author{
Von \\ Wilhelm Groß in Wien $\dagger$.
}

Im folgenden will ich einige ergänzende Bemerkungen meinen vor kurzem erschienenen funktionentheoretischen Arbeiten ${ }^{1,2}$ ) anfügen. Zuerst bringe ich noch einige Sätze über den inneren Häufungsbereich, den ich in $\left.{ }^{2}\right)$ definiert habe, der aber schon in vielen funktionentheoretischen Untersuchungen vorkommt ${ }^{3}$ ), vor allem in den Arbeiten von Study ${ }^{4}$ ) und insbesonders von Lindelöf ${ }^{5,6}$ ). Ich mache hierbei teilweise von den Resultaten und Methoden der letzten, mir in der Zwischenzeit bekannt gewordenen Arbeit $\left.{ }^{8}\right)$ Gebrauch. Ich werde hierdurch in Stand gesetzt, einige wohl ziemlich weit reichende Sätze, die sich auf die Begriffe: innerer Häufungsbereich, Hauptpunkt und deren Beziehungen erstrecken, zu beweisen; ich verweise diesbezüglich insbesonders auf die Sätze von 5., 10., 11.

Zweitens untersuche ich hier das Verhalten von $|w-A|$ bei der Annäherung von $w$ an den Randpunkt $A$, sowie ich in ${ }^{2}$ ) das Verhalten von ampl $(w-A)$ untersucht habe.

*) Kurz nach Erledigung der Korrektur dieser Arbeit ist der Verfasser, Dr. Wilhelm GroB, a. o. Professor an der. Wiener Universität, am 22. Oktober im Alter von 33 Jahren einer schweren Grippenerkrankung erlegen. Die mathematische Welt verliert in ihm einen vielversprechenden jungen Forscher.

Die Schriftleitung.

1) Wilhelm GroB, Uber die Singularitäten analytischer Funktionen; Monatsh. f. Math. u. Phys. XXIX (1918), S. 3-47.

2) Wilhelm Groß, Untersuchungen über die Singularitäten analytischer Funktionen, Math. Zeitschr. II (1918), S. 242-294.

3) Zum Beispiel bei den Untersuchungen über den Abelschen Satz usf.

4) E. Ștudy, Vorlesungen über ausgewählte Gegenstände der Geometrie, 2. Heft, Konforme Abbildung einfach zusammenhängender Bereiche, herausgegeben unter Mitwirkung von W. Blaschke, B. G. Teubner, Leipzig 1913.

5) E. Lindelöf, Mémoire sur certąines inégalites dans la théorie des fonctions monogènes, Acta soc. se. fenn. Bd. XXXV', Nr. 7, 1908.

6) E. Lindelöf, Sur un principe général de l'analyse et ses applications à la théorie de la répresentation conforme, Acta soc. sc. fenn. Bd. XLVI, Nr. 4, 1915. 
Schließlich gebe ich ein Beispiel einer im Einheitskreis meromorphen Funktion, deren absoluter Betrag auf einer Folge von Kreisen $k_{n}$, die gegen den Einheitskreis konvergieren, mit $\frac{1}{n}$ gegen Null geht, während die Funktion nicht identisch verschwindet. Hieraus ergibt sich, daß die Funktion in dem bei der Randzuordnung viel verwendeten Schwarzschen Satze, der von Koebe ${ }^{7}$ ) und mir $^{1}$ ) erweitert wurde, notwendig gewissen Einschränkungen - z. B. sie läßt drei Werte aus - unterworfen sein muß.

\section{Der innere Häufungsbereich.}

1. E. Lindelöf hat auf die einfachste Weise ${ }^{8}$ ) in ${ }^{8}$ ) folgenden Satz bewiesen:

$f(z)$ sei eine innerhalb des Bereiches $T$

$$
\varphi_{1} \leqq \varphi \leqq \varphi_{2}, \quad 0 \leqq r \leqq R
$$

beschränkte und reguläre Funktion von $z=a+r e^{i \varphi}$. Sie möge auf dem in $T$ gegen a strebenden Jordanweg $L$ gegen a konvergieren. $L$ kann mit den Randstrahlen von T Punkte gemein haben, wenn $f(z)$ in diesen Punkten stetig ist.

Dann strebt $f(z)$, wie klein auch $\varepsilon$ sei, in dem Winkel

$$
\varphi_{1}+\varepsilon \leqq \varphi \leqq \varphi_{2}-\varepsilon
$$

für $r \rightarrow 0$ gleichmäßig gegen $\alpha$.

Wird $L$ von einem Randstrahl etwa von $\varphi=\varphi_{1}$ gebildet, so geht $f(z)$ für $r \rightarrow 0$ in dem Winkel $\varphi_{1} \leqq \varphi \leqq \varphi_{2}-\varepsilon$ gleichmäßig gegen $\kappa$.

2: Ist nun $f(z)$ eine im Einheitskreise meromorphe Funktion, so habe ich in $\left.{ }^{2}\right)$ als inneren Häufungsbereich von $f(z)$ im Randpunkte $a$ die Vereinigungsmenge der Häufungsbereiche all jener in $a$ endenden Wege definiert, die den Einheitskreis in weiterem Sinne nicht berühren, $d . h$. deren jeder schließlich in einem von $a$ ausgehenden Winkelraum verläuft, dessen Schenkel bei $a$ in den Kreis eintreten. Mit Hilfe dieses Begriffes können wir aus obigem Satze folgende Sätze ableiten:

7) P. Ko ebe, Ränderzuordnung bei konformer Abbildung, Gött. Nachr. 1913, S. 286.

8) Der dem Beweise zugrunde liegende Kern wurde außer von Lindelöf selbst [Sur la représentation conforme C. R. Bd. 158 (1914), S. 245-257] bereits ron anderen Mathematikern benutzt, so von Painlevé, Sur les lignes singulières des fonctions analytiques, Annales de Toulouse Bd. II (1888), S. B 1-B 130; Zoretti, Leçons sur le prolongement analytique, Gauthier Villars 1911; Koebe, Abhandlungen zur Theorie der konformen Abbildung I., Journ. f. Math. Bd. 145 (1914), S. 177-223; Wilhelm GroB, Zur Theorie der Differentialgleichungen mit festcn kritizchen Punkten, Math. Ann. LXXVIII (1918), S. 332-342. 
Ist die Funktion $f(z)$ im Einheitskreis meromorph und läßt sie in der Umgebung der Randstelle a ein Kontinuum von Werten aus, so besteht der innere Haufungsbereich in a aus dem Punkte $\alpha$, falls $f(z)$ auf. irgendeinem Jordanwege, der im Einheitskreise gegen a strebt, gegen . konvergiert.

Wir brauchen hierzu nur durch eine passende Quadratwurzeloperation zu einer Funktion $\varphi$ überzugehen, die in der Umgebung von $a$ im Einheitskreis analytisch und beschränkt ist.

3. Ist $f(z)$ in der Umgebung der Stelle $a$ ausnahmsverzweigt ${ }^{9}$ ), so liefert die Verwendung der dieser Verzweigung zugehörigen Transzendenten den Satz:

Ist $f(z)$ im Einheitskreis meromorph und in der Umgebung der Randstelle a ausnahmsverzweigt, so besteht der innere Häufungsbereich in a aus dem Punkte $\alpha$, wenn $f(z)$ auf irgendeinem Jordanwege, der im Einheitskreise gegen a strebt, gegen a konvergiert, vorausgesetzt, daß $\alpha$ nicht etwa eine Windungsstelle unendlich hoher Ordnung der Ausnahmsverzweigung ist.

4. Die Arbeit ${ }^{5}$ ) von Lindelöł liefert hierzu folgende Ergänzung, die dort allerdings nur für den Fall ausgesprochen ist, daß $f(z)$ drei Werte ausläßt. Sie läßt sich aber auch in der hier gegebenen allgemeineren Form fast mit den gleichen Worten beweisen:

Ist $f(z)$ im Einheitskreis meromorph und in der Umgebung der Randstelle a ausnahmsverzweigt, so besteht der innere Häufungsbereich in a aus dem Punkte a, wenn a eine Windungsstelle unendlich hoher Ausnahmsverzweigung ist und wenn es dabei einen den Einheitskreis in weiterem Sinne nicht berïhrenden Jordanweg des Kreises gibt, der in a mündet und auf dem $f(z)$ gegen c konvergiert.

5. Wir werden nunmehr nachweisen, daß in diesem Satze die Bedingung, daß der in $a$ endende Jordaneinschnitt des Kreises den Einheitskreis in weiterem Sinne nicht berühren darf, überflüssig ist und daher der Satz von 3. folgende Gestalt annimmt:

Ist $f(z)$ im Einheitskreis meromorph und in der Umgebung der Randstelle a ausnahmsverzweigt, so besteht der innere Häufungshereich in $a$ aus dem Punkte $\alpha$, wenn $f(z)$ auf irgendeinem Jordanwege, der im Einheitskreise gegen a strebt, gegen a konvergiert.

6. Wir weisen zuerst folgenden Hilfssatz nach:

Ist $U$ ein im Einheitskreise positives Potential, das auf einem in a mündenden Jordaneinschnitt $W$ des Kreises gegen $\infty$ geht, so geht $U$

9) Vgl. ${ }^{1)}$ S. 33 und 42. 
auf jedem Jordanweg des Kreises gegen $\infty$, der in a mündet hond den Kreis in weiterem Sinne'nicht berührt.

Um allen Schwierigkeiten zu entgehen, ersetzen wir den Kreis durch eine Jordankurve, die ihn in $a$ berührt, sonst ganz im Innern des Kreises gelegen ist und $W$ mit Ausnahme des Punktes $a$ im Innern enthält. Wir bilden das Innere dieser Jorda nkurve $\Re$ auf den Einheitskreis ab. $U$ geht hierbei in ein positives Potential $U^{*}$ des neuen Einheitskreises $K^{*}$ ab, das am Rande mit Ausnahme des Punktes $a^{*}$ überall stetig ist. $W$ geht in einen Jordan einschnitt $W^{*}$ von $K^{*}$ über, der in dem dem Punkte $a$ entsprechenden Punkte $a^{*}$ endet. Auf $W^{*}$ geht $U^{*}$ gegen $\infty$.

Sodann gestalten wir den Jordaneinschnitt $W^{*}$ zu einem Jordanquerschnitt $\bar{W}^{*}$ von $K^{*}$ um, der auch anderseits gegen einen Randpunkt von $K^{*}$ strebt. Dadurch zerfällt $K^{*}$ in zwei Teile $K_{1}^{*}$, bzw. $K_{2}^{*}$. Im Punkte $a^{*}$ bestimmen wir die gegen ihn aus dem Innern von $K^{*}$ zielenden Strahlen durch den Winkel $\varphi(0<\varphi<\pi)$, den sie mit der positiv gerichteten Tangente bilden. Sei nun $K_{1}^{*}$ der Teil, der an den Randteil $\varphi=0$ von $K^{*}$ im Punkte $a$ anschließt. Ich bilde dann $K_{1}^{*}$ konform auf den Einheitskreis $K^{* *}$ ab. Im Punkte $a^{* *}$, der $a^{*}$ entspricht, zähle ich die Winkel der gegen $a^{* *}$ aus dem Innern von $K^{* *}$ zielenden Strahler wie oben.

$U^{* *}$, das Bildpotential von $U^{*}$, ist im Innern regulär und am Rande von $K^{* *}$ mit Ausnahme des Punktes $a^{* *}$ stetig und ist, wie man leicht zeigt, als positives Potential gegeben durch das Poissonsche Integral

$$
U_{(r, \varphi)}^{* *}=\frac{1}{2 \pi} \int_{0}^{2 \pi} U_{(1, \psi)}^{* *} \frac{1-r^{2}}{1+r^{8}-2 r \cos (\varphi-\psi)} d \psi
$$

und da $U_{(1, \psi)}^{* *}$ auf dem Randteile $\varphi=\pi$ beim Herangehen an $a^{* *}$ über alle Grenzen wächst, so zeigen die bekannten Abschätzungsformeln für das Poissonsche Integral, daß $U_{(r, \varphi)}^{* *}$ in dem dem Kreise $K^{* *}$ angehörigen Winkelraum

$$
\delta \leqq \varphi \leqq \pi
$$

gleichmäßig gegen Unendlich geht, wie klein auch $\delta$ gewählt sei.

Nun folgt aus den von mir in ") III. angestellten Betrachtungen, daß die Teile von $K_{1}^{*}$, für die

$$
2 \delta \leqq \varphi \leqq \pi, \quad\left|z^{*}-a^{*}\right|<r^{*},
$$

in dem Teile des Winkelraumes (4) ihr Bild haben, für den $\left|z^{* *}-a^{* *}\right|<r^{* *}$, wobei $r^{* *}$ mit $r^{*}$ gegen Null geht, sofern $r^{*}$ nur genügend klein gewählt ist. Berücksichtigen wir das $K_{2}^{*}$ betreffende analoge Ergebnis, so sehen wir, $\operatorname{da} B U^{*}$ in dem Winkelraum 


$$
2 \delta \leqq \varphi \leqq \pi-2 \delta
$$

bei der Annäherung an $a^{*}$ gleichmäßig gegen Unendlich geht, wobei $\delta$ beliebig klein gewählt werden kann. Berücksichtigen wir die Winkelproportionalität der Abbildung von $\Re$ auf $K^{*}$, so folgt daraus unser Hilfssatz.

7. Die Funktion $f(z)$ sei nun $\mathrm{m}$ Einheitskreis meromorph und lasse etwa in der Umgebung von $a$ drei Werte aus, gegen deren einen $f(z)$ auf dem in $a$ mündenden Jorda neinschnitt $W$ konvergiert. - Die übrigen in Betracht kommenden Fälle der Ausnahmsverzweigung erledigen sich ganz gleichartig. - Es schadet der Allgemeinheit der Betrachtung nichts, wenn wir annehmen, dabs die drei Ausnahmswerte 0,1 und $\infty$ sind und daß $f(z)$ auf $W$ gegen $\infty$ geht.

Es sei nun $\boldsymbol{\nu}(\zeta)$ die Modultranszendente, die die universelle Uberlagerungsfläche über der in den Punkten $0,1, \infty$ punktierten $\zeta$-Ebene auf die obere Halbebene der $w$-Ebene abbildet, und zwar moge die Abbildung so normiert sein, daß dem Punkte $\infty$ der $w$-Halbebene ein Punkt $\propto$ der $\zeta$-Ebene entspricht. Es sei dann der im Einheitskreis der $z$-Ebene reguläre und eindeutige Zweig $v(f(z))$ so bestimmt, daß $v(f(z))$ auf $W$ gegen $\infty$ geht. Der Imaginärteil der Funktion $\nu(f(z))$ ist ein im Einheitskreis reguläres positives Potential, das auf $W$ gegen Unendlich geht, da ja $f(z)$ gegen Unendlich geht. Daher geht $\mathfrak{\Im}(\nu(f(z)))$ auf jedem in a mündenden Jordanquerschnitt des Einheitskreises, der diesen in weiterem Sinne nicht berührt, gegen Unendlich. Daraus folgt aber einmal, daß auf jedem : solchen Wege $v(f(z))$ gegen Unendlich geht, weiter aber auch, daß $f(z)$. selbst auf diesen Wegen gegen Unendlich geht, da $\Im(\nu(f(z)))$ und $f(z)$ gleichzeitig miteinander gegen Unendlich gehen. Damit haben wir aber den'Satz von 4. ohne den einschränkenden Zusatz für den Weg $W$ nachgewiesen und können daher den einfachen allgemeinen Satz von 5. aussprechen.

8. Ich will aber gleich betonen, da $\beta$ in diesen Fällen der Punkt $\alpha$, der den inneren Häufungsbereich des Punktes $a$ bildet, keineswegs Hauptpunkt zu sein braucht, d. h. daB es sehr wohl in $a$ mündende Jordaneinschnitte geben kann, auf denen $f(z)$ a nicht einmal approximiert. Ein einfaches Beispiel hierfür ist

$$
f(z)=e^{\frac{z+1}{z-1}}
$$

Auf jedem gegen den Punkt $z=1$ strebenden Weg des Einheitskreises, der den Einheitskreis in weiterem Sinne nicht berührt, geht $f(z)$ gegen Null; diese stellt daher den inneren Häufungsbereich dar. Auf dem Kreise

$$
\mathfrak{R}\left(\frac{z+1}{z-1}\right)=a<0,
$$


der den Einheitskreis im Punkte 1 berïhrt, nimmt $f(z)$ aber nur Werte an, deren absoluter Betrag $e^{\alpha}$ ist.

Entsprechend bei dem wie oben normierten $v(z)$. Ist $\mu(w)$ die $z u-$ gehörige Umkehrungsfunktion, so geht $\mu(w)$ auf jedem Wege gegen Unendlich, für den $\mathfrak{S}(w)$ gegen Unendlich geht. Daher ist $\infty$ der innere Häufungsbereich der Funktion $\mu(w)$ im Punkte $\infty$. Gehe ich aber auf dem Wege $\Im(w)=\beta>0$ gegen den Punkt $\infty$, so besitzt dieser Weg als Häufungsbereich eine Kurve, die ganz im Endlichen gelegen ist und die Punkte Null und Eins im Innern enthält.

9. Weitere Aussagen können wir jedoch machen, wenn die Funktion in der Umgebung des Punktes $a$ ein Kontinuum von Werten ausläßt und der Wert $\alpha$, gegen den die Funktion $f(z)$ auf dem in $a$ endenden Jordaneinschnitt konvergiert, diesem Kontinuum angehört. Wir können nämlich sagen:

Ist die Funktion $f(z)$ im Einheitskreis meromorph und läßt sie in der Umgebung der Randstelle a ein Kontinuum von Werten aus, so ist der diesem Kontinuum angehörige Wert a ein Hauptpunkt für $f(z)$ in a, wenn $f(z)$ auf irgendeinem in a mündenden Jordaneinschnitt gegen $\alpha$ konvergiert.

Wir betrachten auf der Riemannschen Fläche über der $w$-Ebene die zusammenhängenden Punktmengen $|w-\alpha|=\varrho$ für genügend kleine $\varrho$, die mit dem dem obigen Jorda n einschnitt $W$ entsprechenden Wege $\mathfrak{B}$ Punkte gemein haben. Wir betrachten hierbei nur jenen Teil der Riemannschen Fläche, der einer Umgebung $|z-a| \leqq R$ entspricht, in der $f(z)$ das im Satze angeführte Kontinuum von Werten ausläßt. Ist daher $\varrho$ genügend klein, so hat jede der eingangs erwähnten Punktmengen $|w-\alpha|=\varrho$ eine Länge höchstens gleich $2 \pi \varrho$ und überdeckt die $w$-Ebene nur einfach.

Ich nehme nun auf $W$ einen Punkt $P$ an, für den $|z-a|=R$ und $|f(z)-\alpha|=\mathrm{P} \neq 0$. Nehme ich nun die Gesamtheit der Kreisbögen her, für die $|w-\alpha|=\varrho<P$ ist und die mit $\mathfrak{B}$ Punkte gemein haben. Durch sie wird der Punkt $\alpha$, bzw. der an $\alpha$ stoßende Teil von $\mathfrak{B}$ von $\operatorname{dem} P$ entsprechenden Punkte $Q$ getrennt. Jedem dieser Kreisbögen entspricht ein Querschnitt des Kreiszweiecks $|z-a|=R$, der beiderseits gegen bestimmte Punkte konvergiert $\left.{ }^{10}\right)$. Durch die Gesamtheit dieser Querschnitte wird der Punkt $a$ vom Punkte $P$ getrennt. Daher gibt es zumindest einen Querschnitt darunter, $\operatorname{der} a$ von $P$ trennt, und wir wählen jetzt den ersten dieser Querschnitte, wenn wir von $a$ auf $\operatorname{dem}$ Wege $W$ ausgehen. Bezeichnen wir die durch diese Auswahl für alle $\varrho<\varrho_{1}$ erhaltene Punktmenge auf der Riemannschen Fläche mit $M\left(\varrho_{1}\right)$ und die auf der

10) Vgl. ${ }^{2}$ ) S. 36 . 
Riemannschen Fläche eindeutige Umkehrungsfunktion von $f(z)$ mit $\varphi(w)$, so können wir wieder die Ungleichung verwerten:

$$
\left(\iint_{M\left(\varrho_{1}\right)}\left|\varphi^{\prime}(w)\right| \varrho d \varrho d \varphi\right)^{2} \leqq \bar{\iint}_{M\left(\varrho_{2}\right)} \varrho d \varrho d \varphi \iint_{M\left(\varrho_{1}\right)}\left|\varphi^{\prime}(w)\right|^{2} \varrho d \varrho d \varphi .
$$

Wir haben einmal

Anderseits ist

$$
\iint_{M\left(\varrho_{2}\right)} \varrho d \varrho d \varphi \leqq \varrho_{1}^{2} \pi
$$

$$
\lim _{\varrho_{1}=0} \bar{\iint}_{M\left(\varrho_{1}\right)}\left|\varphi^{\prime}(w)\right|^{2} \varrho d \varrho d \varphi=0
$$

und daraus folgt, daß

$$
\left\lfloor\underline{\lim } \int\left|\varphi^{\prime}(w)\right| \varrho d \varphi=0 .\right.
$$

Es gibt also eine Holge von Querschnitten, die $a$ und $P$ trennen, deren Länge gegen Null geht und auf denen $|f(z)-\alpha|$ mit $\frac{1}{n}$ gegen Null geht. Da jeder Weg, der gegen $a$ strebt, diese Querschnitte passieren muß, ist a Hauptpunkt.

10. Wir können aber noch einen weitergehenden Satz als einfache Folgerung der Verallgemeinerungen des Picardschen Theorems nachweisen:

Ist die Funktion $f(z)$ im Einheitskreis meromorph, konvergiert sie auf dem gegen den Randpunkt a strebenden Jordaneinschnitt $W$ des Kreises gegen $\alpha$ und ist a Häufungspunkt einer Menge von Werten, die in einer bestimmten Umgebung von a nur endlich oft angenommen werden, so ist a Hauptpunkt von $f(z)$ in a.

$\mathrm{E}_{\mathbb{S}}$ sei bemerkt, $\mathrm{da} ß \alpha$ selbst in der Umgebung von $a$ unendlich oft. angenommen werden darf. - Nehmen wir nun an, es gebe einen gegen $a$ strebenden Weg $\bar{W}$, dessen Häufungsbereich $a$ nicht enthalte. Es gibt daher ein Komplementärgebiet $\gamma$ des Häufungsbereiches von $\bar{W}$, das $\alpha$ enthält, und daher müßte $f(z)$ in jeder Umgebung von $a$ - und zwar schon zwischen $W$ und $\bar{W}$ - jeden Wert von $\gamma$ mit Ausnahme von höchstens zwei unendlich oft annehmen im Widerspruch damit, daß $a$ Häufungspunkt einer Menge von Werten ist, die in einer gewissen Umgebung von $a$ nur endlich of $t$ angenommen werden,

Eine unmittelbare Folge hiervon ist der Satz:

Ist die Funktion $f(z)$ im Einheitskreis meromorph, ist ihr Häufungsbereich im Randpunkte a nirgends dicht und konvergiert sie auf einem Jordaneinschnitt des Kreises, der in a mündet, gegen den Wert a, so ist a Hauptpunkt.

Weiter:

Ist die Funktion $f(z)$ im Einheitskreis meromorph und konvergiert 
sie auf einem in a mündenden $J$ ordaneinschnitt des $K$ reises gegen einen Randpunkt a des Häufungsbereiches von $f(z)$ in a, so ist a Hauptpunkt.

11. Nunmehr wollen wir aber mit Benutzung der entsprechend abgeänderten Lindelöf schen Methode von $\left.{ }^{6}\right)$ S. 30 ff. folgenden ziemlich weitgehenden Satz nachweisen ${ }^{11}$ ):

Ist die Funktion $f(z)$ im Einheitskreis meromorph und besitzt. sie auf dem in a mündenden Jordan einschnitt $W$, der den Kreis in weiterem Sinne nicht berührt, einen Häufungswert $\alpha$, der Häufungspunkt einer Menge $M$ von Werten ist, die in einer bestimmten Umgebung von a nur endlich oft angenommen werden, so ist a Hauptpunkt von $f(z)$ in a

Der Fall, daß $f(z)$ auf irgendeinem in $a$ mündenden Jordanquerschnitt gegen a konvergiert, ist durch die vorhergehenden Sätze schon erledigt. Wir nehmen daher an, daß es keinen derartigen Weg gibt. Ohne Schaden der Allgemeinheit können wir annehmen, daß $\alpha$ einen endlichen Wert, etwa den Wert Null besitzt und daB $f(z)$ in einer bestimmten Umgebung $U$ von $a:|z-a|<R$ regulär ist; denn wir können durch eine lineare Transformation von $w=f(z)$ a nach Null und einen Punkt von $M$ nach Unendlich verlegen. Wir setzen weiter voraus, daß von $f(z)$ in $U$ die beiden Werte $A$ und $B$ nicht angenommen werden urd daß $W$ ganz in $U$ liegt.

Es sei nun $\lambda$ der kleinere der Werte $\mid A ;$ und $|B|$. Wir bezeichnen die $f(z)$ in $U$ entsprechende Riemannsche Fläche mit $\Re$, das Bild von $W$ auf $\Re$ mit $\mathfrak{B S}$. Es sei $P$ ein Punkt von $W$, für dessen Bild $Q$ auf $\Re$ $Q \mid<i$ gilt. Ich wähle nun, indem ich auf $\mathfrak{B}$ so fortschreite, daß der Bildpunkt auf $W$ von $P$ gegen $a$ wandert, eine unendliche Folge von Punkten $G_{i}$ :

$$
|Q|>\left|G_{i}\right|>\left|G_{i+1}\right| . \quad\left|G_{i}\right| \rightarrow 0,
$$

deren Bildpunkte gegen $a$ konvergieren. Anderseits sei $S_{1}, S_{2}, \ldots, S_{n}, \ldots$ eine gegen Null konvergierende Folge von Werten

$$
|Q|>\left|S_{n}\right|>\left|S_{n+1}\right| \rightarrow 0,
$$

die in der Umgebung $U$ von $a$ nur endlich oft angenommen werden.

11) Vor Kenntnisnahme der Lindelöfschen Arbeit ${ }^{\circ}$ ) hatte ich mit den Methoden von $\left.{ }^{2}\right)$ II. vorliegenden Satz unter den beiden engeren Annahmen bewiesen:

1. daß $\propto$ Randpunkt eines der durch den Häufungsbereich von $f(z)$ in $\alpha$ bestimmten Komplementärgebiete ist,

2. $\mathrm{da} B \alpha$ einem Kontinuum von Werten angehöre, die in der Umgebung von a höchstens $N$-mal angenommen werden.

Aus 1 hatte ich bereits die Sätze von 20. sbgeleitet. 
12. Ich betrachte jene zusammenhängenden Teile von $\Re$, für die $|w|<\left|S_{n}\right|$; in denen Punkte $G_{i}$ liegen. Ist $n$ genügend groß; so muß es deren unendlich viele geben. Gäbe es nämlich eine Folge von $n$ :

$$
n_{1}, n_{\Delta}, \ldots, n_{i}, \ldots,
$$

so daß für jede dieser Zahlen nur endlich viele zusammenhängende Teile $|w|<\left|S_{n_{i}}\right|$ bestünden, in denen Punkte $G$ lägen, so gäbe es einmal einen $n_{1}$ entsprechenden Teil $t_{n_{1}}$, in dem unendlich viele Punkte von (6) lägen; von den endlich vielen Teilen, die $n_{2}$ entsprechen, sind einige Teile von $t_{n_{1}}$ und in einem von ihnen $t_{n_{2}}{ }^{\prime}$ müßten wieder unendlich viel Punkte unserer Folge liegen. Fahren wir so fort, so erhalten wir eine Folge einander enthaltender Riemannscher Bereiche; $t_{n_{1}}, t_{n_{2}}, \ldots$, deren jeder un. endlich viel Punkte unserer Folge enthält.

Wähle ich nun in jedem dieșer Bereiche je einen sunkt von (6):

$$
G_{i_{1}}, G_{i_{2}}, \ldots G_{i_{k}}, \ldots
$$

so daß alle diese Punkte voneinander verschieden sind, so kann ich $G_{i_{1}}$ mit $G_{i_{2}}$ in $t_{n_{1}}$ durch einen Jordanweg verbinden, $G_{i_{3}}$ mit $G_{i_{3}}$ in $t_{n_{2}}$ durch einen Jordanweg, der den $G_{i_{2}}$ und $G_{i_{2}}$ verbindenden Weg nicht trifft. und indem ich so fortfahre, erhalte ich auf $\Re$ einen Jordanweg, der ein Einschnitt von $\Re$ ist, denn er geht durch die Punkte $G_{i_{1}}, \ldots, G_{i_{k}}$, deren Bilder gegen $a$ konvergieren. Da die Projektion dieses Einschnittes gegen den Punkt 0 strebt, so strebt das Bild dieses Einschnittes nach $\left.{ }^{1}\right)$ S. 36 gegen einen Randpunkt von $U$, und zwar müßte dieser Randpunkt $a$ sein, da das Bild des Einschnittes durch die gegen $a$ konvergierenden Bilder der Punkte $G_{i_{1}}, \ldots$ geht im Widersprach damit, daß es keinen in $a$ mündenden Jordaneinschnitt gibt, auf $\operatorname{dem} f(z)$ gegen Null konvergiert.

Wir sehen gleichzeitig, dals, wenn wir ein festes genügend großes $n$ annehmen, eine Folge aus den $G$ derart heransgegriffen werden kann:

$$
Q_{1}, Q_{2}, \ldots, Q_{n}, \ldots ;\left|Q_{n}\right|>\left|Q_{n+1}\right| ;: Q_{n} \mid \rightarrow 0,
$$

deren Bildpunkte gegen $a$ streben, so da $\beta$ es einen und hur einen zusammenhängenden Teil $T_{i}$ von $\Re:|w|<\left|S_{n}\right|$ gibt, in dem der Punkt $Q_{i}$ und nur dieser liegt.

13. Es gibt nun einen und nur einen $T_{i}$ begrenzenden Querschnitt bzw. Rückkehrschnitt $q_{i}$ von $\Re$, der $T_{i}$ vom Punkte $Q$ trennt; für ihn ist $|w|=\left|S_{n}\right|$. Da über dem Punkte $S_{n}$ nur endlich viel $-m$ - Punkte von $\Re$ liegen, so konvergiert der Querschnitt $q_{i}$ - von den höchstens $m$ Rückkehrschnitten abgesehen - beiderseits gegen bestimmte Punkte und daher konvergiert auch das Bild $x_{i}$ dieses Querschnittes nach ${ }^{1}$ ) S. 36 beiderseits gegen bestimmte von $a$ verschiedene Randpunkte von $U$. 
Ich ziehe dann von $Q$ auf $\Re$ einen Halbstrahl gegen das Unendliche, dessen Verlängerung nach der anderen Seite durch Null geht. Diesem Einschnitt von $\Re$ entspricht ein Einschnitt von $U$, der keinen der Querschnitte $x_{i}$ trifft und der nach ${ }^{1}$ ) S. 36 gegen einen bestimmten Randpunkt $b \neq a$ von $U$ konvergiert. Der Rand von $U$ zerfällt so durch $a$ und $b$ in zwei Teile, die ich entsprechend dem positiven Umlaufssinn als links und rechts von $a$ bezeichne.

Ich bemerke zuerst, daß die Häufungspunkte der Querschnitte $x_{i}$ auf dem Rande von $U$ liegen; ist $c$ irgendein innerer Punkt von $U$ und entspricht ihm auf $\mathfrak{R}$ eine $(m-1)$-fache Windungsstelle, so läßt sich um $c$ eine Umgebung derart abgrenzen, daß sie höchstens von $m \varkappa_{i}$ durchsetzt wird.

Nehmen wir nun an, daß die Endpunkte jedes einzelnen von unendlich vielen der $x_{i}$ beiderseits von $a$ liegen, so müßte daher jeder gegen $a$ strebende Jordaneinschnitt von $U$ unendlich viele dieser $x_{i}$ durchsetzen, und da auf diesen $x_{i}$ die Beziehung gilt:

$$
|w|=\left|\boldsymbol{S}_{n}\right|
$$

so besäße jeder dieser Wege unter seinen Häufungswerten zumindest einen, für den die Beziehung (9) gilt.

Ist der Nullpunkt kein Hauptpunkt für $f(z)$ in $a-$ und diese Voraussetzung wollen wir vorläufig machen und dann als widerspruchsvoll nachweisen -, so muß es einen Weg geben, der in $a$ mündet und dessen Häufungsbereich vom Nullpunkt einen endlichen Abstand $h$ besitzt. Ist $n$ so groß gewählt, daß

$$
\left|\mathbb{S}_{n}\right|<h
$$

- und dies sei der Fall -, so kann es nur endlich viele $\varkappa_{i}$ geben, deren Endpunkte beiderseits von $a$ liegen.

14. Ich betrachte im folgenden nur solche $x_{i}$ - und alle $x_{i}$ von einem bestimmten Index an gehören dazu -, deren Endpunkte für jeden einzelnen auf einer Seite von $a$ liegen. Nehme ich einen bestimmten dieser Querschnitte her, so besitzt er einen von Null verschiedenen $\mathrm{Ab}$ stand von $a$. Daher können in dem von $\varkappa_{i}$ abgegrenzten Teilgebiet von $U$, das den Punkt $P$ nicht enthält, nur endlich viel Bilder der Punkte $Q$ und daher auch nur endlich viel $x$ liegen.

Anderseits kann $x_{i}$ nur durch endlich viele $x$ von $P$ getrennt werden, da sonst die $x_{i}$ einen Häufungspunkt im Innern von $U$ haben müßten.

Daher läßt sich aus den $x$ eine unendliche Folge mit wachsenden Indizes herausgreifen - ich bezeichne sie in dieser Folge mit

$$
x_{1}^{*}, x_{2}^{*}, \ldots, x_{h}^{*}, \ldots
$$

Bo daß kein $x^{*}$ dieser Folge durch einen andern Querschnitt der Folge 
von $P$ getrennt wird. Durch $x_{h}^{*}$ wird das Bild des Bereiches $T_{h}^{*}$ mit dem Bilde $P_{h}^{*}$ von $Q_{h}^{*}$ von $P$ getrennt.

15. Ich bilde jetzt einmal den Bereich $U$ auf den Einheitskreis der $\zeta$-Ebene $a b$, so daß den Punkten $a$ und $b$ die Punkte +1 und -1 entsprechen. Dem Einschnitt $W$ entspricht hierbei ein Einschnitt $\bar{W}$, der im Punkte +1 mündet, ohne den Einheitskreis zu berühren. $\bar{W}$ liegt daher in der Kreissichel, die von den Kreisen des Büschels durch +1 und -1 gebildet wird, die mit der reellen Achse die Winkel $\frac{\pi}{2}+\eta$ und $\frac{\pi}{2}-\eta$ bilden. In diesem $Z$ weieck liegen daher auch die Bilder der Punkte $P_{h}^{*}$.

Sodann bilde ich den Einheitskreis der $\zeta$-Ebene - und damit $U$ durch

$$
u=\log \frac{\zeta-1}{\zeta+1}
$$

auf den Parallelstreifen der $u$-Ebene:

$$
\frac{\pi}{2}>\mathfrak{\Im}(u)>-\frac{\pi}{2}
$$

ab. Die Funktion $f(z)$ in $u$ geht in die Funktion $\psi(u)$ ir (11) über. Unsere Kreissichel geht dabei in den Parallelstreifen

$$
\frac{\pi}{2}-\eta>\mathfrak{S}(u)>-\frac{\pi}{2}+\eta
$$

über. Wir bestimmen $p$ als kleinste ganze Zahl, die der Bedingung genügt:

$$
\left(\frac{2}{3}\right)^{p} \pi \leqq \eta
$$

16. Es sei $\mathfrak{I}_{i}$ der von dem Bilde $\bar{x}_{i}$ von $x_{i}^{*}$ begrenzte endliche Teilbereich des Parallelstreifens. Da $\mathfrak{I}_{i}$ das Bild $\bar{P}_{i}$ von $P_{i}^{*}$ enthält, so hat $\mathfrak{T}_{i}$ mit dem Parallelstreifen (12) Punkte gemein. Ist $\varphi(u)$ eine Funktion, die in $\mathfrak{I}_{i}$ und auf $\bar{\varkappa}_{i}$ in (11) regulär und absolut kleiner als eins ist, während auf $\bar{x}_{i}$ die Ungleichung bestebt:

$$
|\varphi(u)|<\varepsilon,
$$

so zeigen wir einmal mit Lindelöf, daß $\varphi_{i}(u)$ in den Punkten, die $\mathfrak{T}_{i}$ init dem Parallelstreifen (12) gemein hat, der Ungleichung genügt

$$
|\varphi(u)|<\varepsilon^{\frac{1}{3 p}}
$$

$\bar{x}_{i}$ möge seine Endpunkte auf der Geraden $\mathfrak{S}(u)=-\frac{\pi}{2}$ haben. Das Maximum von $\Im(u): m-\frac{\pi}{2}$ auf $x_{i}$ liegt zwischen $\frac{\pi}{2}$ und $-\frac{\pi}{2}+\eta$. Ien betrachte nun die Punkte von $\mathfrak{T}_{i}$ für die $\Im(u)=\frac{2}{3} m-\frac{\pi}{2}$. Sei $u_{0}$ einer dieser Punkte. Die Funktion 


$$
\Phi(u)=\varphi(u) \cdot \varphi\left(u_{0}+e^{\frac{2 \pi i}{3}}\left(u-u_{0}\right)\right) \cdot \varphi\left(u_{0}+e^{\frac{4 \pi i}{3}}\left(u-u_{0}\right)\right)
$$

ist als reguläre Funktion in einem $u_{0}$ enthaltenden Bereich $\mathfrak{B}$ definiert, der das $u_{0}$ enthaltende Durchschnittsgebiet ist, das der Bereich $\mathfrak{I}_{i}$ mit den beiden Bereichen gemein hat, die man erhält, wenn man $\mathfrak{T}_{i}$ um $u_{0}$ um $120^{\circ}$ bzw. $240^{\circ}$ dreht. $D a ß$ die Berandung von $B$ nur aus Teilen von $\bar{x}_{i}$ und den aus $\bar{x}_{i}$ durch die Drehung entstehenden Linien besteht, sieht man am einfachsten, wenn man bedenkt, daß $\mathfrak{B}$ ganz in dem gleichseitigen Dreieck enthalten ist, dessen Mittelpunkt $u_{0}$ ist und dessen eine Seite in der Geraden $\Im(u)=m-\frac{\pi}{2}$ liegt.

In jedem Randpunkt von $\mathfrak{B}$ mit Ausnahme von höchstens dreien den möglicherweise auftretenden Eckpunkten des erwähnten gleichseitigen Dreiecks - sind die Faktoren von $\Phi(u)$ regulär. Einer von ihnen ist absolut kleiner als $\varepsilon$, während die beiden anderen Faktoren absolut kleiner als eins sind. $\quad \mathrm{Da}$ in $\mathfrak{B}$ obendrein $|\Phi(u)|<1$, so folgt

$$
\left|\varphi\left(u_{0}\right)\right|=\left|\sqrt[3]{\Phi\left(u_{0}\right)}\right|<\varepsilon^{\frac{1}{3}} .
$$

Da $\varphi(u)$ für die Punkte von $\mathfrak{T}_{i}$, für die $\mathfrak{\Im}(u)=\frac{2}{3} m-\frac{\pi}{2}$, absolut kleiner als $\varepsilon^{\frac{1}{3}}$ ist, so ist sie es für alle Punkte von $\mathfrak{T}_{i}$, für die

$$
\mathfrak{\Im}(u) \geqq \frac{2}{3} m-\frac{\pi}{2},
$$

bzw. für alle jene Teilbereiche von $\mathfrak{T}_{i}$, deren Begrenzung nur von Punkte von $\bar{x}_{i}$ und der Geraden $\mathfrak{S}(u)=\frac{2}{3} m-\frac{\pi}{2}$ gebildet wird.

Wenden wir diese Schlußweise auf den Teilbereich von $\mathfrak{T}_{i}$ an, für den $\mathfrak{J}(u)<\frac{2}{3} m-\frac{\pi}{2}$ ist und der an die Gerade $\mathfrak{\Im}(u)=-\frac{\pi}{2}$ stößt, bedenken wir hierbei, $\mathrm{da}$ in den Randpunkten dieses Bereiches, soweit sie nicht auf den Geraden $\Im(u)=-\frac{\pi}{2}$ liegen, $\varphi(u)$ regulär und absolut kleiner als $\varepsilon^{\frac{1}{3}}$ ist, so erkennen wir, daß in allen Teilbereichen von $\mathfrak{T}_{i}$, die nur vor. Punkten von $\bar{x}_{i}$, bzw. der Geraden $\mathfrak{J}(u)=\left(\frac{2}{3}\right)^{2} m-\frac{\pi}{2}$ begrenzt werden, also sicher für alle Punkte von $\mathfrak{T}_{i}$, fürr die $\mathfrak{J}(u) \geqq\left(\frac{2}{3}\right)^{2} m-\frac{\pi}{2}$

$$
|\varphi(u)|<\varepsilon^{\frac{1}{3^{2}}} .
$$

Schließen wir so weiter, so folgt, daß für alle Punkte von $\mathfrak{T}_{\boldsymbol{i}}$, für die $\Im(u) \geqq\left(\frac{2}{3}\right)^{p} m-\frac{\pi}{2}$

$$
|\varphi(u)|<\varepsilon^{\frac{1}{3 p}}
$$


also sicher für alle Punkte von $\mathfrak{I}_{i}$, für die

$$
\mathfrak{J}(u) \geqq-\frac{\pi}{2}+\eta
$$

also für die, die in dem Streifen (12) liegen.

17. $\mathrm{Zu}$ derartigen Funktionen $\varphi_{i}(u)$ kommen wir aber in folgender Weise. Wir bilden einmal die universelle Uberlagerungsfläche der in den Punkten $A, B, \infty$ punktierten $w$-Ebene konform auf den Kreis $|v|<\frac{3}{4}$ der $v$-Ebene ab, so daß dem über Null liegenden Punkte eines bestimmten Blattes der Punkt Null der $v$-Ebene entspricht. Denke ich mir den Kreis durch $S_{n}:|w|=\left|S_{n}\right|$ und die Punkte $Q_{1}^{*}, \ldots, Q_{n}^{*}, \ldots, S_{n} \ldots S_{N}, \ldots$ auf dieses Blatt übertragen, so entspricht einmal dem Kreise in der $v$-Ebene eine geschlossene doppelpunktfreie analytische Kurve $\Re$, die den Nullpunkt umschließt, da ja die Verzweigungsstellen der Uberlagerungsfläche außerhalb des Kreises liegen. In $\Re$ liegen dann die Punkte

$$
K_{1}, \ldots, K_{n} \rightarrow 0, \quad \Sigma_{n}, \ldots, \Sigma_{N} \rightarrow 0,
$$

die den Punkten $Q^{*}$ bzw. $S$ entsprechen.

Auf $\Re$ haben wir einmal

$$
r_{1}<|v|<r_{2}<1,
$$

während die Schwankung des Arguments von $v$ bei einmaligem Durchlaufen von $\Re<\Psi$ ist. Ist $\Sigma_{N},-\left|\Sigma_{N}\right|<\frac{1}{4},-$ nur nahe genug an dem Nullpunkt gelegen, also für $N \geqq N_{1}$, so hat man bei vorgegebenem $\delta<\frac{r_{1}}{2}$, $<\frac{1-r_{2}}{2}$

$$
r_{1}-\delta<\left|v-\Sigma_{N}\right|<r_{2}+\delta<1 \text {. }
$$

während die Schwankung des Arguments von $v-\Sigma_{N}$ bei einmaligem Durchlaufen von $\AA<\Psi+\delta$ ist. Wir können daher unabhängig von $N$ für $N \geqq N_{1}$ eine ganze Zahl $g$ so bestimmen, daß auf $\Omega$

$$
1-\varepsilon_{1}<\left|\sqrt{v-\Sigma_{N}}\right|<1 \text {, }
$$

während die Schwankung des Arguments von $\sqrt[b]{v-\Sigma_{N}}$ bei einmaligem Durchlaufen von $\mathfrak{\Re}<\frac{1}{2 \pi} \varepsilon_{1}$ ist. Gleichzeitig kann ich nach Festsetzung von $g \Sigma_{N}$ so nahe an Null und $j$ so groß wählen, daß

$$
\left|\sqrt[b]{K_{i}-\Sigma_{N}}\right|<\frac{1}{4}, \quad \imath \geqq j .
$$

$\varepsilon_{1}$ wähle ich nun gleich $\left(\frac{1}{4}\right)^{3^{p}} . \quad p$ ist die in (13) bestimmte Zahl. Das obige Ergebnis können wir dann in folgende Worte kleiden: Durchlaufen wir einen Bogen von $\mathfrak{\Re}$, der $\mathfrak{R}$ höchstens einmal überdeckt, so läßt sich ein Punkt $V$ so finden,

$$
1-\left(\frac{1}{4}\right)^{3^{p}}<|V|<1,
$$


daß für einen bestinnten Zweig von $\sqrt{v-\Sigma_{N}}$ die Ungleichung gilt:

$$
\left|\sqrt[b]{v-\Sigma_{N}}-\nabla\right|<\left(\frac{1}{4}\right)^{3^{p}}
$$

Gleichzeitig sei bemerkt, daß im ganzen Kreise $|v|<\frac{3}{4}$ für jede Bestimmung der Wurzel die Ungleichung gilt:

$$
\left|\sqrt{v-\Sigma_{N}}-V\right|<2
$$

18. Es sei nun

$$
v=v(w)
$$

die in 17. verwendete Abbildungsfunktion.

Ich betrachte jetzt ein $\mathfrak{I}_{i}, i \geqq j$, so $\operatorname{da} B(u)$ in $\mathfrak{T}_{i}$ den Wert $\mathbb{S}_{N}$ und auf dem $\mathfrak{T}_{i}$ begrenzenden Querschnitt $\bar{\varkappa}_{i}$ den Wert $S_{n}$ nicht ansimmt; da in (11) nur endlich viele Punkte vorhanden sind, in denen $\psi(u)$ die Werte $S_{N}$ und $S_{n}$ annimmt, so ist eine derartige Wahl stets möglich.

Betrachte ich jetzt die Funktion

$$
\chi(u)=v(\psi(u)),
$$

die in $\mathfrak{T}_{i}$ eindeutig als reguläre Funktion festgelegt ist, wenn wir bestimmen, daß

$$
\chi\left(\bar{P}_{i}\right)=K_{i},
$$

so nimmt $\chi(u)$ in $\mathfrak{T}_{i}$ den $S_{N}$ entsprechenden Wert $\Sigma_{N}$ und auf $\bar{x}_{i}$ den Wert $\Sigma_{n}$ nicht an. Durchläuft $u$ den Querschnitt $\bar{x}_{i}$, so durchläuft $v=\chi(u)$ einen Bogen der Kurve $\Re$, der die Ebene höchstens einmal überdeckt.

Nun ist ein irgendwie fastgelegter $Z$ weig von $\sqrt[b]{\chi(u)-\Sigma_{N}}$ in $\mathfrak{T}_{i}$ eindeutig und es läßt sich nach 17. ein Wert $V$, der der Ungleichung (19) genügt, bestimmen, so daß auf $\ddot{x}_{i}$

$$
\left|\sqrt[b]{\chi(u)-\Sigma_{N}}-V\right|<\left(\frac{1}{4}\right)^{3^{p}} \text {. }
$$

Wir nehmen jetzt als Funktion $\varphi(u)$ von 16. die Funktion

$$
\varphi(u)=\frac{\sqrt[b]{x(u)-\Sigma_{N}}-V}{2} .
$$

Infolge (21) ist in $\mathfrak{T}_{i}|\varphi(u)|<1$ und daher gilt in den Punkten die $\mathfrak{I}_{i}$ mit dem Streifen (11) gemein hat, nach 16.

$$
|\varphi(u)|<\frac{1}{4} \text {. }
$$

Unter diesen Punkten ist aber der Punkt $K_{i}$ enthalten und die Ungleichung (23) würde daher ergeben

$$
|V|<\frac{1}{2}+\left|\sqrt{K_{i}-\Sigma_{N}}\right|<\frac{3}{4}
$$

im Widerspruch zu Ungleichung (19). 
19. Daher ist die Annahme von 13. Ende unrichtig; also ist der Nullpunkt Hauptpunkt und unser Satz ist hiermit bewiesen. Wir können den Satz auch so aussprechen:

Ist a Häufungspunkt einer Menge von Werten, die von der im Einheitskreis meromorphen Funktion in der Umgebung des Randpunktes a nur endlich oft angenommen werden, und gehört a dem inneren Häufungsbereich von $f(z)$ in $a$ an, so ist a Hauptpunkt von $f(z)$ in $a$.

20. Wir fassen hier nur zwei Folgerungen in Sätze:

Ist der Häufungsbereich der im Einheitskreis meromorphen Funktion $w=f(z)$ für den Randpunkt $a$ in dem Gebiete $T$ der $w$-Ebene nirgends dicht, so fällt in $T$ der innere Häufungsbereich mit der Menge der Hauptpunkte zusammen.

Insbesonders:

Ist der Häufungsbereich der im Einheitskreis meromorphen Funktion $f(z)$ für den Randpunkt a nirgends dicht, so fällt für a der innere Häufungsbereich mit der Menge der Hauptpunkte zusammen und besteht aus einem Punkte, bzw. einem Kontinuum.

Es sei noch erwähnt, daß alle Sätze von $\left.{ }^{2}\right)$ II. über den inneren Häufungsbereich aus dem Satze von 11. unmittelbar gefolgert werden können.

\section{Das Verhalten der Abbildung am Rande.}

1. Es sei $f(z)$ eine im Einheitskreise meromorphe Funktion. Dem Randpunkte $a$ möge der erreichbare Randpunkt $\alpha$ der $f(z)$ entsprechenden Riemannschen Fläche $\mathfrak{R}$ entsprechen. Ich will einmal annehmen, daß in einer bestimmten - innerhalb des Einheitskreises genommenen - Umgebung von $a$ der Wert $\alpha$ und der Wert $\infty$ nicht angenommen werde. Dann kann ich in dieser Umgebung $U:|z-a|<R$

$$
\operatorname{ampl}(f(z)-\alpha)=\Im\{\log (f(z)-\alpha)\}
$$

eindeutig festlegen. Ich will nun annehmen, daß dieses Potential in $U$ beschränkt sei.

Ohne die Allgemeinheit der Untersuchung zu beeinträchtigen, können wir $U$ mit dem Einheitskreis zusammenfallen lassen, denn wir können ja stets $U$ auf den Einheitskreis abbilden. Dann läßt sich aber ampl $(f(z)-\alpha)$ im Finheitskreis durch die Formel darstellen:

$$
\operatorname{ampl}(f(z)-\alpha)=\frac{1}{2 \pi} \int_{0}^{2 \pi} G(\psi) \frac{1-r^{8}}{1+r^{2}-2 r \cos (\varphi-\psi)} d \psi,
$$

wobei $G(\psi)$ eine beschränkte, meßbare Funktion von $\psi$ ist, die für alle $\psi$ 
mit Ausnahme höchistens einer Nullmenge definiert ist. Wir haben die rechtsseitigen und linksseitigen Unbestimmtheitsgrenzen von $G(\psi)$ bezüglich der den erreichbaren Punkten von $\mathfrak{\Re}$ entsprechenden Randpunkte des Kreises im Punkte $a:\left(1, \varphi_{0}\right)$ in Betracht gezogen und daraus auf das Verhalten von ampl $(f(z)-\alpha)$ in der Umgebung von $a$ Schlüsse gezogen:

Nunmehr wollen wir aus dem Verhalten von $G(\psi)$ in der Umgebung der Randstelle $a$ auf das Verhalten von $|f(z)-a|$ schließen. Dazu bemerken wir, daB

$$
\log |f(z)-\alpha|=\Re\{\log (f(z)-\alpha)\} .
$$

$\log |f(z)-\alpha|$ ist also das konjugierte Potential zu (24) und wird daher im Einheitskreis durch die Formel gegeben:

$$
\log |f(z)-\alpha|=-\frac{1}{\pi} \int_{0}^{2 \pi} G(\psi) \frac{r \sin (\varphi-\psi)}{1+r^{2}-2 r \cos (\varphi-\psi)} d \psi+C,
$$

wobei $C$ den Wert $\log |f(0)-\alpha|$ besitzt.

2. Es seien nun

$$
o^{++}, u^{+} ; o^{-}, u^{-}
$$

die rechtsseitigen bzw. linksseitigen Unbestimmtheitsgrenzen von $G(\psi)$ im Punkte $a$, diese in obigem Sinne genommen; rechts und links bezeichnen wir nach dem positiven Umlaufssinn des Kreises. Es sei dann

$$
u^{-}-o^{+}=\beta \pi ; \quad u^{+}-o^{-}=\gamma \pi ; \quad \gamma \geqq \beta .
$$

Wir erinnern uns daran, daß einmal

$$
\frac{1}{\pi} \int_{0}^{2 \pi} \frac{r \sin (\varphi-\psi)}{1+r^{2}-2 r \cos (p-\psi)} d \psi=0
$$

und daB

(30) $\log |z-a|=-\frac{1}{2} \int_{0}^{2 \pi} \frac{r \sin (\varphi-\psi) \operatorname{sgn} \sin \left(\varphi_{0}-\psi\right)}{1+r^{2}-2 r \cos (\varphi-\psi)} d \psi+\log |z+a|$.

Wir betrachten sodann die Funktion

$$
\begin{gathered}
=-\frac{1}{\pi} \int_{0}^{8 \pi}\left\{G(\psi)-(\beta-\varepsilon) \frac{\pi}{2} \operatorname{sgn} \sin \left(\varphi_{0}-\psi\right)-\frac{u-+0^{+}}{2}\right\} \frac{r \sin (\psi-\psi)}{1+r^{2}-2 r \cos (\varphi-\psi)} d \psi+C_{1} \\
-(\beta-\varepsilon) \log \mid z+a_{\mid} .
\end{gathered}
$$

Ieh verlege nun durch eine Drehung den Punkt $(1,0)$ in den Punkt $a$ und erstrecke dementsprechend das Integral von $-\pi$ bis $+\pi$. Ich grenze dann ein Intervall $|\psi|<\delta$ so ab, daß 


$$
\begin{aligned}
& \text { für } \quad \delta>\psi>0: u^{+}-\frac{\varepsilon}{2}<G(\psi)<0^{+}+\frac{\varepsilon}{2}, \\
& \text { für }-\delta<\psi<0: 0^{-}+\frac{\varepsilon}{2}>G(\psi)>u^{-}-\frac{\varepsilon}{2} .
\end{aligned}
$$

Bezeichnen wir nun den im Integral von (31) enthaltenen Klammerausdruck mit $\boldsymbol{M}(\psi)$, so ist außerhalb des Intervalles $|\psi|<\delta$, sobald nur $|p|<\frac{\delta}{2}$,

$$
\begin{gathered}
|M(\psi)|<|G(\psi)|+|\beta-\varepsilon| \frac{\pi}{2}+\left|\frac{u^{-}+0^{+}}{2}\right|=L(\psi), \\
1+r^{2}-2 r \cos (\varphi-\psi)>\left(1-\cos \frac{\delta}{2}\right)^{2},
\end{gathered}
$$

daher ist für den von diesem Teil herrührenden Teil des Integrales von (31):

$$
\left|I_{1}\right|<\frac{1}{\pi}\left(1-\cos \frac{\delta}{2}\right)^{-2} \int_{-\pi}^{+\pi} L(\psi) d \psi=C(\delta)
$$

wobei $C(\delta)$ nur von $\delta$ abhängt.

3. Nun gelten für $M(\psi)$, wenn wir etwa annehmen, $\mathrm{da} ß \varphi>0$ ist, folgende Ungleichungen:

$$
\begin{aligned}
& \text { für } \quad-\delta<\psi<0: \quad M(\psi)>\frac{\pi-1}{2} \cdot \varepsilon, \\
& \text { für } \quad \varphi<\psi<\delta: \quad M(\psi)<-\frac{\pi-1}{2} \cdot \varepsilon .
\end{aligned}
$$

Ich bekomme für den Teil des Integrales von (31), für den $|\psi|<\delta$,

$$
\begin{aligned}
I_{2} & <-\frac{\pi-1}{2 \pi} \varepsilon \int_{\delta}^{+\delta} \frac{r \sin (\varphi-\psi) \operatorname{sgn}(-\psi)}{1+r^{2}-2 r \cos (\varphi-\psi)} d \psi \\
& -\frac{1}{\pi} \int_{0}^{\varphi}\left\{M(\psi)+\frac{\pi-1}{2} \varepsilon\right\} \frac{r \sin (\varphi-\psi)}{1+r^{2}-2 r \cos (\varphi-\psi)} d \psi<I_{8}+I_{i} .
\end{aligned}
$$

Dabei habe ich

$$
\begin{aligned}
I_{3} & =-\frac{\pi-1}{2 \pi} \varepsilon \int_{-\pi}^{+\pi} \frac{r \sin (\varphi-\psi) \operatorname{sgn}(-\psi)}{1+r^{2}-2 r \cos (\varphi-\psi)} d \psi+H_{1}(\delta) \\
& =\frac{\pi-1}{\pi} \varepsilon \log |z-a|+H_{3}(\delta)
\end{aligned}
$$

$H_{1}(\delta), H_{2}(\delta)$ sind hierbei Ausdrücke, die absolut genommen kleiner sind als die nur von $\delta$ abhängenden Konstanten $C_{1}(\delta), C_{2}(\delta)$, wenn $|\varphi|<\pi$.

Anderseits habe ich für $0<\psi<\varphi$ :

$$
u^{+}-o^{+}-\varepsilon<M(\psi)+\frac{\pi-1}{2} \varepsilon<0,
$$

also jedenfalls

$$
\left|M(\psi)+\frac{\pi-1}{2} \varepsilon\right|<M .
$$


Daraus folgt

$$
\left|I_{1}\right|<\frac{M}{\pi} \int_{0}^{\varphi} \frac{r \sin (\varphi-\psi)}{1+r^{2}-2 r \cos (\varphi-\psi)} d \psi=\frac{M}{\pi} \log \frac{\sqrt{1+r^{2}-2 r \cos \varphi}}{1-r} .
$$

Wenn wir an den Punkt $(1,0)$ in dem Winkelraum herangehen: $\mid$ ampl $(z-1) \mid<\vartheta$, so bleibt, wenn der Abstand von $(r, \varphi)$ und $(1,0)$ genügend klein ist,

$$
\left.\frac{\sqrt{1+r^{2}-2 r \cos \varphi}}{1-r}<\frac{1}{\cos \vartheta}+\eta^{12}\right) \text {. }
$$

Wir können daher $I_{4}=H_{3}(\delta, \vartheta)$ setzen, wobei $H_{3}(\delta, \vartheta)$ absolut genommen unter der von $\delta, \vartheta$ abhängigen Konstanten $C_{3}(\delta, \vartheta)$ bleibt, sobald $r$ genügend nahe an eins ist.

4. Wir haben daher, wenn $(r, \varphi)$ genügend nahe an $(1,0)$ ist,

$$
D(r, \varphi)<\frac{\pi-1}{-} \varepsilon \log |z-a|+C_{4}(\delta, \vartheta),
$$

wobei die Konstante $C_{4}(\delta, \vartheta)$ nur von $\delta, \vartheta$ abhängt; also

$$
\frac{|f(z)-\alpha|}{|z-a|^{\beta-\varepsilon}}<C_{\mathrm{b}}(\delta, \vartheta)|z-a|^{\frac{\pi-1}{\pi}} \rightarrow 0,
$$

d. h. wie klein ich auch $\varepsilon$ wähle, so geht der Quotien

$$
\frac{|f(z)-\alpha|}{|z-a|^{\beta-z}}, \quad \beta=\frac{u^{-}-o^{+}}{\pi}
$$

gleichmäßig gegen $N u l l$, wenn ich mich aem Punkte a in einem Winkelraum nähere, dessen Schenkel den Einheitskreis nicht berühren.

Ganz entsprechend beweisen wir den partiellen Satz:

Wie klein ich auch $\varepsilon$ wähle, so geht der Quotient

$$
\frac{|f(z)-\alpha|}{|z-a|^{\gamma+\varepsilon}}, \quad \gamma=\frac{u^{+}-o^{-}}{\pi}
$$

gleichmäßig gegen Unendlich, wenn ich mich dem Punkte a in einem Winkelraume nähere, dessen Schenkel den Einheitskreis nicht berühren.

12) Am leichtesten sieht man es folgendermaßen ein: Ich betrachte die Punkte $Q=(1,0), P=(r, \varphi), P_{0}=(r, 0)$ und den dem Punkte $Q$ nächsten Punkt $P_{1}$, in dem der Kreis $|z|=r$ die Gerade schneidet, die unter dem Winkel $\pi-\vartheta$ die ree!le Achse im Punkte 1 trifft. Wir haben

$$
\frac{\sqrt{1+r^{2}-2 r \cos \varphi}}{1-r}=\frac{\overline{P Q}}{\overline{P_{n} Q}}=\frac{\sin \Varangle\left(P P_{0} Q\right)}{\sin \Varangle\left(Q P P_{0}\right)}<\frac{\sin \Varangle\left(P_{1} P_{0} Q\right)}{\sin \Varangle\left(Q P_{1} P_{0}\right)} .
$$

Der letzte Quotient strebt aber für $r \rightarrow 1$ gegen $\frac{1}{\sin \left(\frac{\pi}{2}-\vartheta\right)}=\frac{1}{\cos \vartheta}$. 
Es sei betont, daß hierbei $\beta$ sehr wohl auch negativ sein kann, während $\gamma$ stets größer oder gleich Null ist.

Von besonderem Interesse ist der Fall, wo der Bereich $\Re$ im Punkte $九$ eine rechte, bzw. linke Halbtangente, ev. in dem in $\left.{ }^{2}\right)$ III. erläuterten weiteren Sinne besitzt, so da $\beta=\gamma$ ist und im Punkte $\alpha$ eine Ecke vom Offnungswinkel $\beta$ vorliegt.

Besitzt der einfach zusammenhängende Riemannsche Bereich $\Re$ eine Ecke a vom Offnungswinkel $\beta$ und entspricht dieser bei der durch $w=f(z)$ gelieferten Abbildung von $\mathfrak{\Re}$ auf den Einheitskreis der $z$-Ebene der Punkt a, so gehen, wie klein ich auch $\varepsilon$ wähle, die Quotienten

$$
\frac{|f(z)-\alpha|}{|z-a|^{\beta-\delta}} \text { und } \frac{|f(z)-\alpha|}{|z-a|^{\beta+\varepsilon}}
$$

gleichmäßig gegen Null, bzw. gegen Unendlich, wenn ich mich dem Punkte a in einem Winkelraume nähere, dessen Schenkel den Einheitskreis nicht beriihren.

\section{II. Ein Beispiel zu einem Schwarzschen Satze.}

1. Aus dem Spiegelungsprinzip von $\operatorname{Schwarz}{ }^{12}$ ) folgt unmittelbar folgender Satz:

Ist $F(z)$ eine oberhalb eines Stückes $a . . . b$ der Achse des Reellen in einem gewissen anschließenden Flächenstreifen regulär erklärte analytische Funktion von der Eigenschaft, bei der Annäherung an die Achse des Reellen gleichmäßig einem konstanten Wert zuzustreben, so reduziert sich diese Funktion überhaupt auf eine Konstante,

den Koebe $^{7}$ ) formuliert und sodann erweitert hat. Zu erwähnen ist seir eleganter Beweis in einer späteren Arbeit ${ }^{13}$ ). Mit nochmaliger Erweiterung habe ich ${ }^{\mathbf{1}}$ ) dem Satze folgende Form gegeben:

Ist $\boldsymbol{F}(\boldsymbol{z})$ eine oberhalb der Achse des Reellen in dem längs des Stückes $[a, b]$ derselben anschließenden Gebiete $G$ erklärte, eindeutige meromorphe Funktion, die drei Werte ausläßt, gibt es dann eine Folge von Linien $\lambda_{n}$ in $G$, die gegen $[a, b]$ in seiner ganzen Ausdehnung konvergieren, so daß die Funktionswerte auf $\lambda_{n}$ sich von einer Konstante $k_{n}$ um Werte unterscheiden, deren absoluter Betrag kleiner als $\varepsilon_{n}$ ist, $\lim _{n=\infty} \varepsilon_{n}=0$,

12) H. A. Schwarz, Zur Integration der partiellen Differentialgl. $\frac{\partial^{2} u}{\partial x^{8}}+\frac{\partial^{2} u}{\partial y^{2}}=0$ unter vorgeschriebenen Grenz- und Unstetigkeitsbedingungen, Berl. Monatsh. 1870, Ges. Abh., Bd. II, S. $175-210$.

13) P. Koebe, Abhandlungen zur Theorie der konformen Abbildung I, Journ. f. Math. 145 (1915), S. 177-223. 
bzw. ihrem absoluten Betrag nach über $g_{n}$ liegen, wobei $g_{n}$ mit $n$ gegen Unendlich geht, so kann $F(z)$ nicht von einer Konstanten verschieden sein.

Die Bedingung, daß $F(z)$ in $G$ drei Werte ausläßt, kann man durch die allgemeinere Bedingung ersetzen, daß die der Funktion $w=F(z)$ in $G$ entsprechende Riemannsche Fläche über der $w$-Ebene ausnahmsverzweigt ist. \&usnahmsverzweigt bezeichne ich eine Riemannsche Fläche dann, wenn es drei, bzw. vier, bzw. fünf Punkte der $w$-Ebene gibt, so daß alle ijber einem dieser Punkte $i$ liegenden Stellen der Riemannschen Fläche, von endlich vielen abgesehen, Windungsstellen von einer Ordnung sind, die um eins vermehrt durch $m_{i}$ teilbar ist, wobei $\frac{1}{m_{1}}+\frac{1}{m_{2}}+\frac{1}{m_{3}}<k$, bzw. $\frac{1}{m_{1}}+\frac{1}{m_{2}}+\frac{1}{m_{3}}+\frac{1}{m_{4}}<2$, bzw. $\frac{1}{m_{1}}+\frac{1}{m_{2}}+\frac{1}{m_{3}}+\frac{1}{m_{4}}+\frac{1}{m_{5}}<3$ ist. Die letzte dieser Ungleichungen legt den $m_{i}$, die ja $\geqq 2$ sind, keine Bedingung auf. Liegen über einem Punkt nur endlich viel Blätter der Riemannschen Fläche, so können wir dem $m_{i}$ den Wert $\propto$ erteilen.

2. Es erhebt sich jedoch die Frage, ob es überhaupt notwendig ist, die in $G$ eindeutige meromorphe Funktion Einschränkungen zu unterwerfen. Daß diese Frage zu bejahen ist, zeige ich durch Konstruktion einer im Einheitskreis meromorphen Funktion $f(z)$, für die es eine Reihe konzentrischer, gegen den Einheitskreis konvergierender. Kreise $k_{1}, \ldots, k_{n}, \ldots$ gibt, so da $\beta$ der absolute Betrag von $f(z)$ auf $k_{n}$ kleiner als $\frac{1}{2^{n}}$ ist. Da das konstruierte $f(z)$ im Nullpunkt einen Pol besitzt, ist es sicher von einer Konstanten verschieden.

3. Zur Konstruktion benutzen wir folgende Hilfsbetrachtung: Ist $p(z)$ eine auf und außer dem Kreise $K$ regulär-analytische Funktion, die im Unendlichen verschwindet, so wird durch das Integral $\Phi(z)=-\frac{1}{2 \pi i} \int_{\boldsymbol{K}} \frac{\varphi(\zeta) d \zeta}{\zeta-z}$ im Außengebiet von $K \varphi(z)$, im Innengebiet hingegen Null dargestellt. Ist dann $*$ ein abgeschlossener Kreisring, der zu $K$ konzentrisch ist und $K$ in seinem Innern enthält, so können wir $\Phi(z)$ derart durch eine Riemannsche Summe

$$
\Psi(z)=\sum_{i}^{n} \frac{\varphi\left(\zeta_{i}\right) \zeta_{1}}{z-\zeta_{i}} \frac{1}{n}
$$

ersetzen, so dal $|\Phi(z)-\Psi(z)|$ in den beiden durch $x$ bestimmten Komplementärgebieten kleiner ist als das beliebig vorgegebene $\varepsilon$, so daß sich also $\Psi^{\prime}(z)$ in dem äußeren Komplementärgebiet von $\varphi(z)$ im inneren Komplementärgebiet von Null um weniger als $\varepsilon$, absolut genommen, unter- 
scheidet $\left.{ }^{14}\right) . \Psi(z)$ ist hierbei eine in der ganzen Ebene eindeutige, meromorphe Funktion, die im Unendlichen verschwindet.

4. Wir betrachten einmal die Kreise um den Nullpunkt $k_{1}, \ldots, k_{n}, \ldots$, von denen $k_{n}$ den Radius $1-\frac{1}{2 n+1}$ besitzt, sodann die Kreise um den Nullpunkt: $k_{1}^{*}, \ldots, k_{n}^{*}, \ldots$, von denen $k_{n}^{*}$ den Radius $1-\frac{1}{2 n}$ besitzt. $k_{n}^{*}$ betten wir in einen Kreisring $\varkappa_{n}$, der begrenzt wird durch die Kreise von den Radien $1-\frac{1}{2 n}-\frac{1}{8 n^{2}}$ und $1-\frac{1}{2 n}+\frac{1}{8 n^{2}}$. Die Kreise $k_{n}$ liegen sämtlich außerhalb der abgeschlossenen Kreisringe $x_{n}$.

Ich gehe sodann von der Funktion $\frac{1}{z}$ aus und konstruiere mir nach 3., indem ich $k_{1}^{*}$ als $K, x_{1}$ als $\varkappa$ nehme, eine meromorphe Funktion $\psi_{1}(z)$, die sich in dem inneren Komplementärgebiet von $\varkappa_{1}$ von Null, in dem äußeren Komplementärgebiet von $\varkappa_{1}$ von $\frac{1}{z}$ um weniger als $\frac{1}{2^{2}}$, absolut genommen, unterscheidet. Entsprechend konstruiere ich mir nach 3., in-

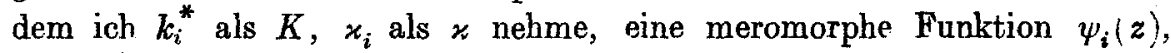
die sich in dem inneren Komplementärgebiet von $\varkappa_{i}$ von Null, in dem äußeren Komplementärgebiet von $x_{i}$ von $\frac{1}{z}-\sum_{1}^{i-1} \psi_{h}(z)$ um weniger als $\frac{1}{2^{i+1}}$, absolut genommen, unterscheidet.

Die Funktion

$$
G(z)=\frac{1}{z}-\sum_{1}^{\infty} \psi_{h}(z)
$$

ist die gewünschte Funktion.

5. $G(z)$ ist im Einheitskreis eindeutig und meromorph, denn ist $\bar{z}$ ein Punkt aus $x_{n}$; so haben wir

$$
\left|\frac{1}{z}-\sum_{1}^{\infty} \psi_{h}(\bar{z})+\psi_{n}(\bar{z})\right| \leqq\left|\frac{1}{\bar{z}}-\sum_{1}^{n-1} \psi_{h}(\bar{z})\right|+\sum_{n+1}^{\infty} h\left|\psi_{h}(\bar{z})\right|
$$

also für $n=1$

$$
\leqq\left|\frac{1}{\bar{z}}\right|+\sum_{2}^{\infty} \frac{1}{2^{h+1}}=\left|\frac{1}{z}\right|+\frac{1}{4},
$$

während für $n>1$ gilt

$$
\leqq \frac{1}{2^{n}}+\sum_{n+1}^{\infty} \frac{1}{2^{h+1}}<\frac{1}{2^{n-1}}
$$

14) Vgl. C. Runge, Zur Theorie der eindeutigen analytischen Funktionen, Acta math. VI (1885), S. $229-244$. 
64 Wilhelm Groß. Verhalten dex konformen Abbildung am Rande.

Liegt hingegen $\bar{z} \mathrm{im}$ Innenkomplementärgebiet von $x_{1}$, so ist

$$
\left|\sum_{1}^{\infty} h \psi_{h}(\bar{z})\right| \leqq \sum_{1}^{\infty} h\left|\psi_{h}(\bar{z})\right|<\sum_{1}^{\infty} h \frac{1}{2^{h+1}}=\frac{1}{2} .
$$

Zwischen $x_{n}$ und $x_{n+1}$ haben wir

daher

$$
\left|\frac{1}{z}-\sum_{1}^{n} \psi_{h}(\bar{z})\right|<\frac{1}{2^{n+1}},
$$

$$
\begin{aligned}
& \left|\frac{1}{z}-\sum_{1}^{\infty} \psi_{h}(\bar{z})\right| \leqq\left|\frac{1}{\bar{z}}-\sum_{1}^{n} \psi_{h}(\bar{z})\right|+ \\
& +\sum_{n+1}^{\infty}\left|\psi_{h+1}(\bar{z})\right|<\frac{1}{2^{n+1}}+\sum_{n+1}^{\infty} \frac{1}{2^{n+1}}=\frac{1}{2^{n}} .
\end{aligned}
$$

$G(z)$ besitzt daher als Pole den Punkt Null und die abzählbar vielen Pole der Funktionen $\psi_{h}(z)$, deren Häufungspunkte auf dem Einheitskreis liegen. Nach (39) gilt auf $k_{n}$, welcher Kreis ja zwischen $x_{n}$ und $x_{n+1}$ liegt, die Beziehung

$$
|G(z)|<\frac{1}{2^{n}}
$$

(Ejngegangen am 28. Juli 1918.) 